\title{
Synthesis of $\alpha, \beta$-Unsaturated Ketones by Rhodium-Catalyzed Carbonylative Arylation of Internal Alkynes with Arylboronic Acids
}

\author{
Melih Kuş, Özge Aksin Artok, Firat Ziyanak, Levent Artok* \\ Department of Chemistry, Faculty of Science, Izmir Institute of Technology, Urla 35430, Izmir, Turkey \\ Fax +90(232)7507509; E-mail: leventartok@iyte.edu.tr \\ Received 5 June 2008
}

\begin{abstract}
The Rh-catalyzed reaction of arylboronic acids with internal alkynes under a $\mathrm{CO}$ atmosphere in the presence of an acid additive afforded $\alpha, \beta$-unsaturated ketones as the major products. Hydroacylation of internal alkynes, except in the case of diaryl acetylenes, proceeded in syn fashion, yielding the $E$-configured isomer. A mixture of $E$ - and $Z$-isomers was obtained with diphenyl acetylene. Reactions were also highly regioselective for various nonsymmetric alkynes.
\end{abstract}

Key words: carbonylation, boron, rhodium, enones, alkynes

We have shown recently that carbonylation of an arylboronic acid and internal alkyne mixture in the presence of a rhodium complex can yield the $\alpha, \beta$-unsaturated ketone $\mathbf{3}$, 5-aryl-2(5H)-furanone $\mathbf{4}$, indenone $\mathbf{5}$, and indanone $\mathbf{6}$ products, and we have successfully optimized the method to generate 4 with excellent selectivity (Scheme 1 ). ${ }^{1}$ In addition, we also want to switch the selectivity of the process to the side of the other products, as they are also highvalue reagents.

Carbonylative coupling of arylboronic acids with unsaturated substrates is believed to proceed through an acylrhodation step by an in situ generated acylrhodium complex. ${ }^{1,2}$ During optimization of the reaction conditions for the selective construction of $\mathbf{3}$, we envisioned that prompt protodemetalation of the alkenylrhodium intermediate $\mathbf{C}$, before insertion of $\mathrm{CO}$, which leads to the product 4 , would result in an $\alpha, \beta$-unsaturated ketone 3 (Scheme 2); hence, we considered that the presence of an acidic additive would promote the protodemetalation step, provided that the catalyst retains its activity.

In general, aldehydes are used as acyl-donating reagents in rhodium-catalyzed hydroacylation reactions. Rhodiumcatalyzed acylation reactions generally require challenging $\mathrm{C}-\mathrm{H}$ activation, and acylrhodium intermediates are required to be stabilized by chelation with coordinating functionalities in substrates or in additives, or application

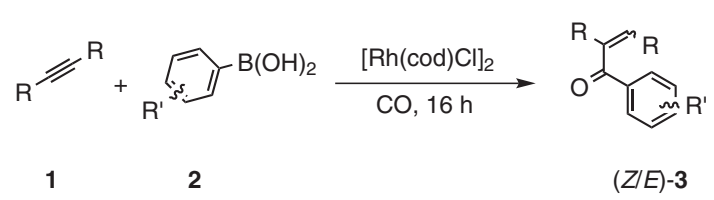

Scheme 1 Carbonylative arylation

SYNLETT 2008, No. 17, pp 2587-2592

Advanced online publication: 10.09 .2008

DOI: 10.1055/s-2008-1078047; Art ID: D20708ST

(c) Georg Thieme Verlag Stuttgart · New York

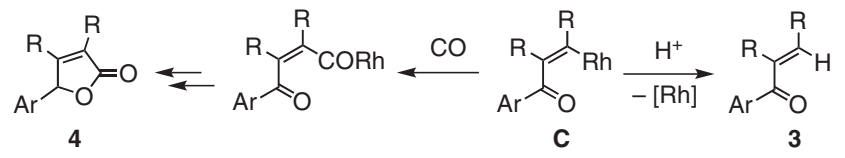

Scheme 2

of high pressure of gaseous olefin or $\mathrm{CO}$ in order to avoid competing decarbonylation reactions. ${ }^{3-5}$

We describe in this report that internal alkynes can also be hydroacylated in the presence of an acid additive, by acylrhodium reagents, which are generated in situ from arylboronic acids under a $\mathrm{CO}$ atmosphere. This reaction offered both atom economy and an environmentally benign process for the synthesis of $\alpha, \beta$-unsaturated ketones containing an aroyl moiety. In the course of this study a rhodium-catalyzed carbonylative addition of arylboronic acids to terminal alkynes was reported, ${ }^{2 \mathrm{~d}}$ wherein alkylsubstituted terminal alkynes were converted to enones with moderate to good yields by the use of excess alkyne. Nevertheless, no example for internal alkynes has been presented, probably owing to a higher tendency of internal alkynes to afford the product type 4 .

The effect of many variables, such as Rh complex, solvent, acid additives, $\mathrm{CO}$ pressure, and temperature on the reaction efficiency were throughly studied. After establishment of the optimum conditions, the scope of the method was examined for a number of arylboronic acids and alkynes. Alkynes (1.5 mmol) and arylboronic acids (3 mmol) were reacted in a methanol-water (9.9:0.1 mL) mixture in the presence of $\left[\mathrm{Rh}\left(\mathrm{C}_{2} \mathrm{H}_{4}\right)_{2} \mathrm{Cl}\right]_{2}(3 \% \mathrm{Rh})$ and 2 mmol of $\mathrm{CF}_{3} \mathrm{COOH}$ under 5 atm of $\mathrm{CO}$ pressure at $80{ }^{\circ} \mathrm{C}$. The reaction of diphenyl acetylene (1a) with several arylboronic acids yielded mixtures of both $Z$ - and $E$-isomers of the corresponding enones $\mathbf{3 a}$ which can be isolated separately by flash chromatography on silica gel (Table 1)

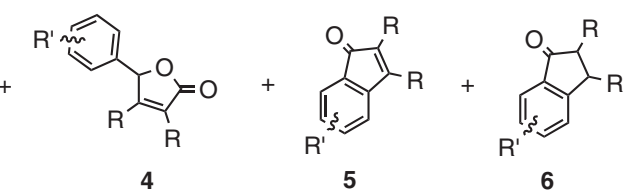

4 
Table 1 The Synthesis of Enones (3a) by Carbonylative Addition of Various Arylboronic Acids (2) to Alkyne 1a

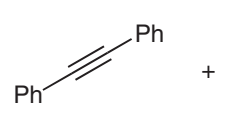

$1 \mathrm{a}$

$1.5 \mathrm{mmol}$

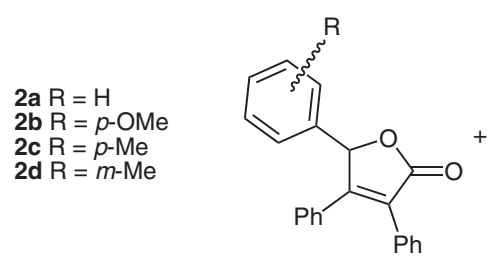

$4 a$

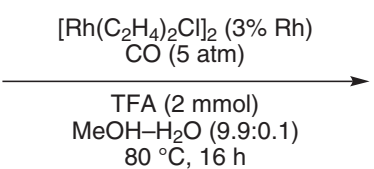

$80^{\circ} \mathrm{C}, 16 \mathrm{~h}$<smiles>O=C(/C(=C/P)c1ccccc1)c1ccccc1</smiles>

$3 a$<smiles>CC(=O)/C(=C\c1ccccc1)c1ccccc1</smiles>

$5 a \quad 6 a \quad 7 a$

\begin{tabular}{|c|c|c|c|c|c|c|}
\hline \multirow[b]{2}{*}{ Entry } & \multirow[b]{2}{*}{2} & \multicolumn{2}{|l|}{ Yield (\%) } & \multirow[b]{2}{*}{$\mathbf{5 a}^{\mathrm{b}}$} & \multirow[b]{2}{*}{$6 a^{b}$} & \multirow[b]{2}{*}{$7 \mathbf{a}^{1}$} \\
\hline & & $3 \mathbf{a}^{\mathrm{a}}$ & $4 a^{b}$ & & & \\
\hline 1 & $2 a$ & $\begin{array}{l}\text { 3aa } \\
(33 Z, 25 E)\end{array}$ & $\begin{array}{l}\mathbf{4 a a} \\
(11)\end{array}$ & $\begin{array}{l}\mathbf{5 a a} \\
(3)\end{array}$ & $\begin{array}{l}\mathbf{6 a a} \\
(12)\end{array}$ & (4) \\
\hline 2 & $2 \mathbf{b}$ & $\begin{array}{l}\text { 3ab } \\
(45 Z, 31 E)\end{array}$ & $\begin{array}{l}\text { 4ab } \\
(5)\end{array}$ & $\begin{array}{l}\mathbf{5 a b} \\
(2)\end{array}$ & $\begin{array}{l}\mathbf{6 a b} \\
(1)\end{array}$ & (3) \\
\hline 3 & $2 c$ & $\begin{array}{l}\text { 3ac } \\
(55 Z, 16 E)\end{array}$ & $\begin{array}{l}\mathbf{4 a c} \\
(8)\end{array}$ & $\begin{array}{l}\mathbf{5 a c} \\
(<1)\end{array}$ & $\begin{array}{l}\mathbf{6 a c} \\
(5)\end{array}$ & (2) \\
\hline 4 & $2 d$ & $\begin{array}{l}\text { 3ad } \\
(29 Z, 32 E)\end{array}$ & $\begin{array}{l}\mathbf{4 a d} \\
(7)\end{array}$ & $\begin{array}{l}\text { 5ad } \\
\text { (3) }\end{array}$ & $\begin{array}{l}\text { 6ad } \\
\text { (9) }\end{array}$ & (5) \\
\hline
\end{tabular}

${ }^{a}$ Isolated product.

${ }^{\mathrm{b}}$ Determined by GC.

along with byproducts furanones $\mathbf{4 a}$, indenones $\mathbf{5 a}$, indanones 6a and a methoxycarbonylated product of diphenyl acetylene, methyl 2,3-diphenylacrylate (7a).

Isomers of 3a were identified or estimated by comparing IR carbonyl frequencies and melting points based on the literature. It has been reported that the existence of steric inhibition of the enone resonance in $Z$-enones results in the appearance of their carbonyl absorption bands at relatively higher wavenumbers than those of the $E$-isomers. ${ }^{7}$

The reaction of arylboronic acid 2a with alkyne 1a afforded the isolated products $(E)$ - and $(Z)-3 \mathbf{a a}$ in overall yield of 58\% and produced byproducts at $30 \%$ yield (Table 1 , entry 1). Arylboronic acids, $\mathbf{2 b}$ and $\mathbf{2 c}$, which have methoxy and methyl groups, respectively, at the para position of the phenyl ring gave the corresponding hydroacylation products in yields of $76 \%$ for both $(E)$ - and $(Z)-3 \mathbf{a b}$ and $70 \%$ yields for both $(E)$ - and $(Z)-3 a c$. Lower amounts of byproducts (11 and $16 \%$, respectively, Table 1 , entries 2 and 3) were generated when using these arylboronic acids. The reaction with $m$-tolylboronic acid (2d) proceeded to give the corresponding enones $(E)$ - and $(Z)-3$ ad in an overall yield of $61 \%$, along with $24 \%$ formation of the byproducts (Table 1 , entry 4 ).

The reactions involving $o$-tolylboronic or $p$-(trifluoromethyl)phenylboronic acids with alkyne 1a resulted in correspondingly low enone yields, remarkably increased amount of side products, and even yielded significant amounts of corresponding hydroarylation products. These results indicate that enone formation is responsive to the sterical and electronic nature of arylboronic acids.

Hydroaroylation proceeded exclusively with syn selectivity for other alkynes, which have only one aryl substituent or none, yielding only the $E$-isomer of the corresponding enones as determined by NOE studies (Table 2). ${ }^{8}$

The carbonylative reaction of 4-octyne (1) with $\mathbf{2 a}$ yielded relatively low isolated yield of $(E)-\mathbf{3 b a}(30 \%$, Table 2 , entry 1$)$. Better yields of $(E)$-3bb and $(E)$-3bc were obtained via the reaction of $\mathbf{1 b}$ and arylboronic acids $\mathbf{2 b}$ and 2c at yields of $57 \%$ and $53 \%$, respectively (Table 2, entries 2 and 3). Reactions were also regioselective for those alkynes that had been activated with an ester functionality. The aroyl group was introduced selectively at the $\beta$ position with respect to the electron-withdrawing group (Table 2, entries 4 and 5). High regioselectivity was also observed with 1-phenylpropyne (1e), the aroyl group primarily introduced at the methyl-substituted acetylenic carbon, giving 3ea and 3eb predominantly upon reaction with the arylboronic acids $\mathbf{2} \mathbf{a}$ and $\mathbf{2 b}$, respectively, which are separable from their regioisomers (Table 2, entries 6 and 7). Nevertheless, the presence of a larger alkyl group on the alkynyl carbon somewhat reduced its regioselectivity, probably due to increased steric hindrance (Table 2, entry 8 ).

The overall yield of byproducts was nearly $30 \%$ for the reaction of $\mathbf{1 b}$ with $\mathbf{2 a}$ mixture, whereas it was less than $10 \%$ for other reactions as determined by GC. 
Table 2 The Synthesis of Enones $\mathbf{3}$ by Carbonylative Addition of Various Arylboronic Acids $\mathbf{2}$ to Alkynes $\mathbf{1}$

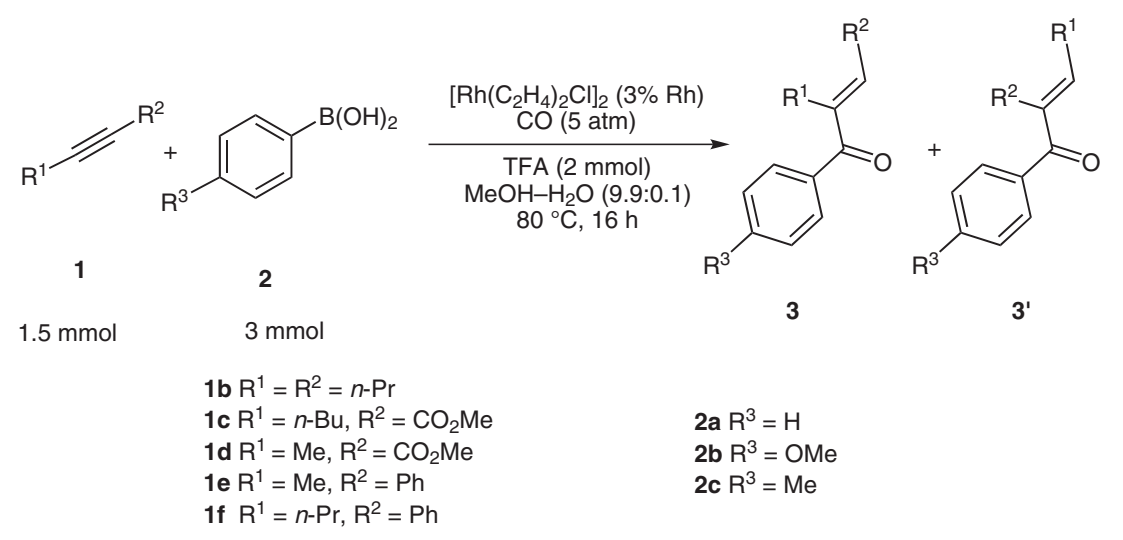

\begin{tabular}{|c|c|c|c|c|}
\hline \multirow[b]{2}{*}{ Entry } & \multirow[b]{2}{*}{1} & \multicolumn{2}{|c|}{ Yield (\%) } & \multirow[b]{2}{*}{$3^{\prime}$} \\
\hline & & 2 & $3^{\mathrm{a}}$ & \\
\hline 1 & $1 b$ & $\mathbf{2 a}$ & 3ba (30) & - \\
\hline 2 & $1 b$ & $2 b$ & $3 \mathbf{b b}(57)$ & - \\
\hline 3 & $1 b$ & $2 c$ & 3bc (53) & - \\
\hline 4 & $1 \mathrm{c}$ & $2 b$ & $3 \mathbf{c b}(64)$ & $(<1)^{\mathrm{b}}$ \\
\hline 5 & 1d & $2 b$ & 3db (54) & $(2)^{\mathrm{b}}$ \\
\hline 6 & $1 e$ & $\mathbf{2 a}$ & 3ea (42) & $(3)^{\mathrm{b}}$ \\
\hline 7 & $1 e$ & $2 b$ & 3eb (74) & $(6)^{b}$ \\
\hline 8 & 1f & $2 b$ & $\mathbf{3 f b}(41)$ & $(13)^{\mathrm{a}}$ \\
\hline
\end{tabular}

${ }^{\mathrm{a}}$ Isolated product.

${ }^{\mathrm{b}}$ Determined by GC.

As for the reaction mechanism, transmetalation of arylboronic acid with the catalyst is probably followed by insertion of $\mathrm{CO}$ into the arylrhodium bond (Scheme 3 ). The resultant aroylrhodium $\mathbf{B}$ undergoes 1,2-addition to the alkyne in a syn fashion to give a vinylrhodium species $\mathbf{C}$, which then should undergo protodemetalation to afford the $E$-enone. Nevertheless, one may consider that exchange of Rh on alkenyl carbon and aryl hydrogen at the ortho position with respect to the carbonyl functionality $\mathbf{D}$ and subsequent protodemetalation of aryl-attached Rh could be an alternative route to the end product in analogy to the Rh-catalyzed hydroarylation of alkynes with arylboronic acids (1,4-shift) as shown by Hayashi et al. ${ }^{9} \mathrm{We}$ eliminated this route by considering the result of the similar reaction of $\mathbf{1 b}$ with $\mathrm{PhB}(\mathrm{OH})_{2}-d_{5}$ under a $\mathrm{CO}$ atmosphere, where indeed no contribution of the proton on the carbonyl-tethered phenyl ring or deuterium on the $\beta$-unsaturated carbon of the related enone product was detected.

A question yet remained as to how the mixture of $E$ - and $Z$-isomers are formed with diphenyl acetylene. An experiment using the pure $E$-isomer of $\mathbf{3 a b}$ under the general reaction protocol, but in the absence of arylboronic acid, brought about an isomerization to the $Z$-configuration in about $25 \%$ yield. Although this result shows that $E$ -

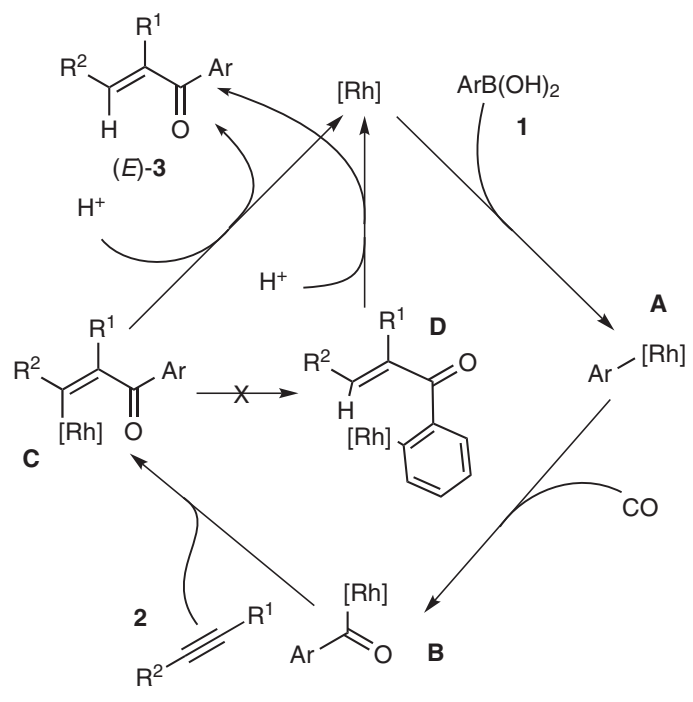

Scheme 3

enones formed from diphenyl acetylene can isomerize partly during the course of the carbonylative arylation reactions, it cannot account for the $Z / E$ ratios given in Table 1. Probably the intermediate $\mathbf{C}$ can also undergo isomerization which is facilitated by the extended conju- 
gation when the alkenyl carbons are substituted with two aryl groups (Scheme 4).<smiles>C=CC(=C(C(=O)Br)c1ccccc1)C(c1ccccc1)c1ccccc1</smiles>

C<smiles></smiles><smiles>O=C(Br)/C(=C\c1ccccc1)c1ccccc1</smiles>

$(Z)-3 a$
Scheme 4

In summary, we have been able to switch the product selectivity of the method involving the rhodium-catalyzed reaction of an arylboronic acid-internal alkyne mixture in a $\mathrm{CO}$ atmosphere in favor of $\alpha, \beta$-unsaturated ketones instead of 5-aryl-2(5H)-furanones. ${ }^{10}$ The methodology established in this study demonstrates an atom economical, relatively mild, and simple manner by which $\alpha, \beta$-unsaturated ketones may be synthesized. Currently, we are focusing our research towards on the refinement and product tunability of the method.

\section{Acknowledgment}

This work was supported by the Scientific and Technical Research Council of Turkey (TBAG-106T385), National Boron Research Institute (BOREN-2006-14-Ç13-09), and IZTECH (2007-IYTE-14). We thank Ms. I. Özçelik for the measurement of NMR spectra.

\section{References and Notes}

(1) Aksin, Ö.; Dege, N.; Artok, L.; Türkmen, H.; Çetinkaya, B. Chem. Commun. 2006, 3187.

(2) (a) Sauthier, M.; Castanet, Y.; Mortreux, A. Chem. Commun. 2004, 1520. (b) Chochois, H.; Sauthier, M.; Maerten, E.; Castanet, Y.; Mortreux, A. Tetrahedron 2006, 62, 11740. (c) Menard, F.; Weise, C. F.; Lautens, M. Org. Lett. 2007, 9, 5335. (d) Dheur, J.; Sauthier, M.; Castanet, Y.; Mortreux, A. Adv. Synth. Catal. 2007, 349, 2499.

(3) (a) Willis, M. C.; Randell-Sly, H. E.; Woodward, R. L.; Steven, J.; McNally, S. J.; Currie, G. S. J. Org. Chem. 2006, 71, 5291. (b) Tanaka, K.; Shibata, Y.; Suda, T.; Hagiwara, Y.; Hirano, M. Org. Lett. 2007, 9, 1215. (c) Imai, M.; Tanaka, M.; Tanaka, K.; Yamamoto, Y.; Imai-Ogata, N.; Shimowatari, M.; Nagumo, S.; Kawahara, N.; Suemune, H. J. Org. Chem. 2004, 69, 1144. (d) Jun, C.-H.; Lee, H.; Hong, J.-B.; Kwon, B. I. Angew. Chem. Int. Ed. 2002, 41, 2146. (e) Moxham, G. L.; Randell-Sly, H. E.; Brayshaw, S. K.; Woodward, R. L.; Weller, A. S.; Willis, M. C. Angew. Chem. Int. Ed. 2006, 45, 7618. (f) Tanaka, K.; Tanaka, M.; Suemune, H. Tetrahedron Lett. 2005, 46, 6053. (g) Willis, M. C.; Sapmaz, S. Chem. Commun. 2001, 2558. (h) Jun, C.-H.; Jo, E.-A.; Park, J.-W. Eur. J. Org. Chem. 2007, 1869. (i) Stemmlera, R. T.; Bolma, C. Adv. Synth. Catal. 2007, 349, 1185. (j) Imai, M.; Tanaka, M.; Nagumo, S.; Kawahara, N.; Suemune, H. J. Org. Chem. 2007, 72, 2543. (k) Rueda, X. Y.; Castillón, S. J. Organomet. Chem. 2007, 692, 1628. (1) Lochow, C. F.; Miller, R. G. J. Am. Chem. Soc. 1976, 98 , 1281. (m) Kokubo, K.; Matsumasa, K.; Miura, M.; Nomura, M. J. Org. Chem. 1997, 62, 4564. (n) Miura, M.; Nomura, M. J. Synth. Org. Chem. Jpn. 2000, 58, 578. (o) Kokubo, K.; Matsumasa, K.; Nishinaka, Y.; Miura, M.; Nomura, M. Bull. Chem. Soc. Jpn. 1999, 72, 303. (p) Marder, T. D.; Roe,
D. C.; Milstein, D. Organometallics 1988, 7, 1451.

(4) Other acylation reagents used: (a) Hydrogenative aroylation with an anhydride: Hong, Y.-T.; Barchuk, A.; Krische, M. J. Angew. Chem. Int. Ed. 2006, 45, 6885.

(b) Hydroacylation of ethylene with acyl halides: Schwartz, J.; Cannon, J. B. J. Am. Chem. Soc. 1974, 96, 4721.

(5) Other metal catalysis - Ir catalyzed: (a) Nishinaka, Y.; Satoh, T.; Miura, M.; Morisaka, H.; Nomura, M.; Matsui, H.; Yamaguchi, C. Bull. Chem. Soc. Jpn. 2001, 74, 1727. Ni catalyzed: (b) Tsuda, T.; Kiyoi, T.; Saegusa, T. J. Org. Chem. 1990, 55, 2555. Ru catalyzed: (c) Kondo, T.; Tsuji, Y.; Watanabe, Y. Tetrahedron Lett. 1987, 28, 6229.

(d) Kondo, T.; Akazome, M.; Tsuji, Y.; Watanabe, Y. J. Org. Chem. 1990, 55, 1286. Co catalyzed: (e) Lenges, C. P.; Brookhart, M. J. Am. Chem. Soc. 1997, 119, 3165.

(6) Typical Conditions for the Synthesis of 3

A mixture of alkyne $(1.5 \mathrm{mmol})$, arylboronic acid $(3 \mathrm{mmol})$, $\left[\mathrm{Rh}\left(\mathrm{C}_{2} \mathrm{H}_{4}\right)_{2} \mathrm{Cl}\right]_{2}(3 \mathrm{~mol} \% \mathrm{Rh})$, TFA $(2 \mathrm{mmol}), \mathrm{H}_{2} \mathrm{O}(0.1 \mathrm{~mL})$ in $\mathrm{MeOH}(9.9 \mathrm{~mL}$, predried) was added into a $50 \mathrm{~mL}$ stainless steel autoclave with a glass insert tube. Then, the sealed autoclave was evacuated, and purged with $10 \mathrm{~atm} C O$ twice, successively. Subsequently, the reactor was pressurized to $5 \mathrm{~atm}$ with $\mathrm{CO}$ and the mixture was stirred magnetically in a preheated oil bath. After cooling, the reaction mixture was recovered with EtOAc. The products were analyzed by GC and GC-MS and isolated by column chromatography. Purity of the products were $>96 \%$ as determined by ${ }^{1} \mathrm{H}$ NMR analyses.

$(E, Z)-1,2,3-T r i p h e n y l p r o p-2-e n-1-o n e ~(3 a a)$

Hexane-EtOAc.

Isomer $(E)$-3aa: white solid; $\mathrm{mp}$ 98.7-99.2 ${ }^{\circ} \mathrm{C}$ (lit.: 99-101 $\left.{ }^{\circ} \mathrm{C},{ }^{7 \mathrm{a}} 103-103.5^{\circ} \mathrm{C},{ }^{7 \mathrm{~b}} 96.5-97.5^{\circ} \mathrm{C}^{7 \mathrm{c}}\right) .{ }^{1} \mathrm{H}$ NMR $(400 \mathrm{MHz}$, $\left.\mathrm{CDCl}_{3}\right): \delta=7.07-7.56(\mathrm{~m}, 14 \mathrm{H}), 7.86(\mathrm{~d}, J=7.2 \mathrm{~Hz}, 2 \mathrm{H})$. ${ }^{13} \mathrm{C} \mathrm{NMR}\left(100 \mathrm{MHz}, \mathrm{CDCl}_{3}\right): \delta=126.4,128.0,128.2,128.4$, 128.7, 128.8, 128.9, 129.7, 130.1, 133.6, 135.4, 136.3, 138.0, 140.8, 199.4. MS (EI): $m / z(\%)=284(93)\left[\mathrm{M}^{+}\right]$, 256(5), 206(9), 178(31), 105(100), 77(20). FTIR (ATR): $v=$ 1649 (CO) (lit.: 1661, $\left.{ }^{\text {7a }} 1640^{7 \mathrm{c}}\right) \mathrm{cm}^{-1}$. HRMS: $\mathrm{m} / \mathrm{z}$ calcd $\left[\mathrm{M}^{+}\right]$: 284.1196; found: 284.1189 .

Isomer (Z)-3aa: yellow solid; mp 78.0-84. ${ }^{\circ} \mathrm{C}$ (lit.: 85-87 $\left.{ }^{\circ} \mathrm{C},{ }^{7 \mathrm{a}} 88-89{ }^{\circ} \mathrm{C},{ }^{7 \mathrm{~b}} 82.5-83.5^{\circ} \mathrm{C}^{7 \mathrm{c}}\right) .{ }^{1} \mathrm{H}$ NMR $(400 \mathrm{MHz}$, $\left.\mathrm{CDCl}_{3}\right): \delta=7.10-7.50(\mathrm{~m}, 14 \mathrm{H}), 7.99(\mathrm{~d}, J=6.8 \mathrm{~Hz}, 2 \mathrm{H})$. ${ }^{13} \mathrm{C} \mathrm{NMR}\left(100 \mathrm{MHz}, \mathrm{CDCl}_{3}\right): \delta=127.9,128.2,128.3,128.8$, $128.9,129.7,129.8,130.3,132.2,134.8,136.5,138.2$, 140.1, 140.8, 197.6. MS (EI): $m / z(\%)=284(87)\left[\mathrm{M}^{+}\right], 256$ (7), 206 (20), 178 (47), 105 (100), 77 (83). FTIR (ATR): $v=1660$ (CO) (lit.: 1671, ${ }^{\text {a }} 1661^{7 \mathrm{c}}$ ) $\mathrm{cm}^{-1}$. HRMS: $\mathrm{m} / \mathrm{z}$ calcd $\left[\mathrm{M}^{+}\right]$: 284.1196; found: 284.1197.

(E,Z)-1-(4-Methoxyphenyl)-2,3-diphenylprop-2-en-1one (3ab)

Hexane-benzene; hexane- $\mathrm{CH}_{2} \mathrm{Cl}_{2}$.

Isomer $(E)$-3ab: white solid; mp 81.1-84.3 (lit.: 84-84.3 $\left.{ }^{\circ} \mathrm{C}^{7 \mathrm{c}}\right) .{ }^{1} \mathrm{H}$ NMR $\left(400 \mathrm{MHz}, \mathrm{CDCl}_{3}\right): \delta=3.85(\mathrm{~s}, 3 \mathrm{H}), 6.92$ $(\mathrm{d}, J=8.8 \mathrm{~Hz}, 2 \mathrm{H}), 7.09-7.36(\mathrm{~m}, 11 \mathrm{H}), 7.90(\mathrm{~d}, J=8.8$ $\mathrm{Hz}, 2 \mathrm{H}) .{ }^{13} \mathrm{C} \mathrm{NMR}\left(100 \mathrm{MHz}, \mathrm{CDCl}_{3}: \delta=55.5,113.6\right.$, 127.9, 128.2, 128.6, 128.8, 129.6, 130.2, 130.4, 132.3, 135.0, 136.8, 137.8, 141.0, 163.1, 196.3. MS (EI): $\mathrm{m} / z(\%)=$ 314 (47) $\left[\mathrm{M}^{+}\right], 197$ (14), 178 (14), 135 (100), 107 (9), 92 (10), 77 (19). FTIR (ATR): $v=1633$ (CO) (lit.: $1640^{7 \mathrm{c}}$ ) $\mathrm{cm}^{-}$ 1. HRMS: $m / z$ calcd $\left[\mathrm{M}^{+}\right]$: 314.1301 ; found: 314.1294 . Isomer (Z)-3ab: pale yellow solid; $\mathrm{mp} 88.7-89.2{ }^{\circ} \mathrm{C}$ (lit.: $\left.78-79{ }^{\circ} \mathrm{C}^{7 \mathrm{c}}\right) .{ }^{1} \mathrm{H} \mathrm{NMR}\left(400 \mathrm{MHz}, \mathrm{CDCl}_{3}\right): \delta=3.79(\mathrm{~s}, 3 \mathrm{H})$, $6.83(\mathrm{~d}, J=8.8 \mathrm{~Hz}, 2 \mathrm{H}), 7.12-7.48(\mathrm{~m}, 11 \mathrm{H}), 7.97(\mathrm{~d}$, $J=8.8 \mathrm{~Hz}, 2 \mathrm{H}) .{ }^{13} \mathrm{C}$ NMR $\left(100 \mathrm{MHz}, \mathrm{CDCl}_{3}\right): \delta=55.4$, 114.0, 126.3, 127.9, 128.1, 128.4, 128.8, 129.4, 129.5, $132.1,135.5,138.2,140.9,164.0,197.9$. MS (EI): $m / z(\%)=$ 
314 (58) $\left[\mathrm{M}^{+}\right], 197$ (18), 178 (19), 135 (100), 107 (12), 92 (13), 77 (23). FTIR (ATR): $v=1657$ (CO) (lit.: $1655^{7 \mathrm{c}}$ ) $\mathrm{cm}^{-1}$. HRMS: $\mathrm{m} / z$ calcd $\left[\mathrm{M}^{+}\right]$: 314.1301 ; found: 314.1301 . $(E, Z)-2,3-D i p h e n y l-1-p$-tolylprop-2-en-1-one (3ac) Hexane-benzene.

Isomer (E)-3ac: white solid; $\mathrm{mp} 90.8-91.8{ }^{\circ} \mathrm{C} .{ }^{1} \mathrm{H}$ NMR $\left(400 \mathrm{MHz}, \mathrm{CDCl}_{3}\right): \delta=2.41(\mathrm{~s}, 3 \mathrm{H}), 7.08-7.39(\mathrm{~m}, 13 \mathrm{H})$, $7.79(\mathrm{~d}, J=8.0 \mathrm{~Hz}, 2 \mathrm{H}) .{ }^{13} \mathrm{C}$ NMR $\left(100 \mathrm{MHz}, \mathrm{CDCl}_{3}\right): \delta=$ 21.6, 126.3, 127.9, 128.2, 128.8, 128.8, 129.0, 129.6, 130.1, $130.3,134.9,135.3,136.7,139.1,140.9,143.0,197.5 . \mathrm{MS}$ (EI): $m / z(\%)=298(75)\left[\mathrm{M}^{+}\right], 283(15), 205(6), 181(23)$, 178 (25), 119 (100), 91 (48). FTIR (ATR): $v=1643$ (CO) $\mathrm{cm}^{-1}$. HRMS: $\mathrm{m} / z$ calcd $\left[\mathrm{M}^{+}\right]: 298.1352 ; 298.1346$. Isomer (Z)-3ac: pale yellow solid; $\mathrm{mp} 59.8-61.8{ }^{\circ} \mathrm{C} .{ }^{1} \mathrm{H}$ $\operatorname{NMR}\left(400 \mathrm{MHz}, \mathrm{CDCl}_{3}\right): \delta=2.32(\mathrm{~s}, 3 \mathrm{H}), 7.10-7.47(\mathrm{~m}$, $13 \mathrm{H}), 7.89$ (d, $J=8.4 \mathrm{~Hz}, 2 \mathrm{H}) .{ }^{13} \mathrm{C}$ NMR $(100 \mathrm{MHz}$, $\left.\mathrm{CDCl}_{3}\right): \delta=21.7,126.3,128.0,128.1,128.5,128.8,129.5$, 129.7, 129.9, 134.0, 135.5, 138.1, 141.0, 144.7, 199.0. MS (EI): $m / z(\%)=298(100)\left[\mathrm{M}^{+}\right], 283(12), 182(20), 119(55)$, 91 (11). FTIR (ATR): $v=1654(\mathrm{CO}) \mathrm{cm}^{-1}$. HRMS: $\mathrm{m} / z$ calcd $\left[\mathrm{M}^{+}\right]$: 298.1352; found: 298.1341.

(E,Z)-2,3-Diphenyl-1-m-tolylprop-2-en-1-one (3ad) Hexane-benzene.

Isomer $(E)$-3ad: further column chromatographed with hexane-EtOAc; white solid; $\mathrm{mp} 66.3-68.7{ }^{\circ} \mathrm{C} .{ }^{1} \mathrm{H}$ NMR $\left(400 \mathrm{MHz}, \mathrm{CDCl}_{3}\right): \delta=2.40(\mathrm{~s}, 3 \mathrm{H}), 7.06-7.38(\mathrm{~m}, 13 \mathrm{H})$, $7.64(\mathrm{~d}, J=7.6 \mathrm{~Hz}, 1 \mathrm{H}), 7.68(\mathrm{~s}, 1 \mathrm{H}) .{ }^{13} \mathrm{C} \mathrm{NMR}(100 \mathrm{MHz}$, $\left.\mathrm{CDCl}_{3}\right): \delta=21.4,127.06,127.9,128.08,128.2,128.8$, 128.9, 129.7, 130.2, 130.3, 132.9 134.9, 136.6, 138.2, 138.2, 140.0, 140.9, 197.9. MS (EI): $\mathrm{m} / \mathrm{z}(\%)=298(75)\left[\mathrm{M}^{+}\right], 283$ (10), 181 (23), 178 (25), 119 (100), 91 (48). FTIR (ATR): $v=1635(\mathrm{CO}) \mathrm{cm}^{-1}$. HRMS: $\mathrm{m} / z$ calcd $\left[\mathrm{M}^{+}\right]: 298.1352$; 298.1351 .

Isomer (Z)-3ad: yellow oil. ${ }^{1} \mathrm{H}$ NMR $\left(400 \mathrm{MHz}, \mathrm{CDCl}_{3}\right)$ : $\delta=2.32(\mathrm{~s}, 3 \mathrm{H}), 7.10-7.48(\mathrm{~m}, 13 \mathrm{H}), 7.65(\mathrm{~d}, J=8.0 \mathrm{~Hz}, 1$ $\mathrm{H}), 7.83(\mathrm{~s}, 1 \mathrm{H}) .{ }^{13} \mathrm{C} \mathrm{NMR}\left(100 \mathrm{MHz}, \mathrm{CDCl}_{3}\right): \delta=21.3$, 126.3, 127.3, 128.0, 128.1, 128.4, 128.6, 128.8, 129.9, $129.9,134.5,135.5,136.3,138.1,138.5,141.0,199.5$. MS (EI): $m / z(\%)=298(92)\left[\mathrm{M}^{+}\right], 283(11), 181(25), 178(30)$, 119 (100), 91 (33). FTIR (ATR): $v=1660(\mathrm{CO}) \mathrm{cm}^{-1}$. HRMS: $m / z$ calcd $\left[\mathrm{M}^{+}\right]$: 298.1352; found: 298.1359 . $(E)$-1-Phenyl-2-propylhex-2-en-1-one (3ba)

Hexane-EtOAc; pale yellow oil. ${ }^{1} \mathrm{H}$ NMR $(400 \mathrm{MHz}$, $\left.\mathrm{CDCl}_{3}\right): \delta=0.94(\mathrm{t}, J=7.4 \mathrm{~Hz}, 3 \mathrm{H}), 0.96(\mathrm{t}, J=7.4 \mathrm{~Hz}, 3$ H), 1.41-1.52 (m, $4 \mathrm{H}), 2.27(\mathrm{q}, J=7.2 \mathrm{~Hz}, 2 \mathrm{H}), 2.47(\mathrm{t}$, $J=7.2 \mathrm{~Hz}, 2 \mathrm{H}), 6.20(\mathrm{t}, J=7.6 \mathrm{~Hz}, 1 \mathrm{H}), 7.38-7.43(\mathrm{~m}, 2$ $\mathrm{H}), 7.47-7.52(\mathrm{~m}, 1 \mathrm{H}), 7.63-7.67(\mathrm{~m}, 2 \mathrm{H}) .{ }^{13} \mathrm{C}$ NMR $(100$ $\left.\mathrm{MHz}, \mathrm{CDCl}_{3}\right): \delta=14.0,14.1,22.2,28.7,30.9,128.0,129.3$, 131.4, 139.1, 141.2, 145.7, 199.0. MS (EI): $m / z(\%)=216$ (100) $\left[\mathrm{M}^{+}\right], 173$ (7), 145 (9), 105 (27), 77 (5). FTIR (ATR): $v=1647(\mathrm{CO}) \mathrm{cm}^{-1}$. HRMS: $\mathrm{m} / \mathrm{z}$ calcd $\left[\mathrm{M}^{+}\right]: 216.1509$; found: 216.1507 .

(E)-1-(4-Methoxyphenyl)-2-propylhex-2-en-1-one (3bb) Hexane-EtOAc; pale yellow oil. ${ }^{1} \mathrm{H}$ NMR $(400 \mathrm{MHz}$, $\left.\mathrm{CDCl}_{3}\right): \delta=0.91-0.98(\mathrm{~m}, 6 \mathrm{H}), 1.41-1.51(\mathrm{~m}, 4 \mathrm{H}), 2.26(\mathrm{q}$, $J=7.2 \mathrm{~Hz}, 2 \mathrm{H}), 2.46(\mathrm{t}, J=7.6 \mathrm{~Hz}, 2 \mathrm{H}), 3.86(\mathrm{~s}, 3 \mathrm{H}), 6.11$ $(\mathrm{t}, J=7.6 \mathrm{~Hz}, 1 \mathrm{H}), 6.91(\mathrm{~d}, J=8.8 \mathrm{~Hz}, 2 \mathrm{H}), 7.71(\mathrm{~d}, J=8.8$ $\mathrm{Hz}, 2 \mathrm{H}) .{ }^{13} \mathrm{C}$ NMR $\left(100 \mathrm{MHz}, \mathrm{CDCl}_{3}\right): \delta=14.0,14.1,22.2$, $22.3,29.2,30.7,55.4,113.3,131.4,131.8,141.1,143.1$, 162.6, 198.0. MS (EI): $m / z(\%)=246(14)\left[\mathrm{M}^{+}\right], 203(22)$, 175 (18), 135 (100), 107 (10), 92 (14), 77 (21). FTIR (ATR): $v=1641(\mathrm{CO}) \mathrm{cm}^{-1}$. HRMS: $\mathrm{m} / z$ calcd $\left[\mathrm{M}^{+}\right]$: 246.1614; found: 246.1603 .

(E)-2-Propyl-1-p-tolylhex-2-en-1-one (3bc)

Hexane-EtOAc; pale yellow oil. ${ }^{1} \mathrm{H}$ NMR $(400 \mathrm{MHz}$, $\left.\mathrm{CDCl}_{3}\right): \delta=0.94(\mathrm{t}, J=7.6 \mathrm{~Hz}, 6 \mathrm{H}), 1.46(\mathrm{sext}, J=7.6 \mathrm{~Hz}$, $4 \mathrm{H}), 2.26(\mathrm{q}, J=7.6 \mathrm{~Hz}, 2 \mathrm{H}), 2.40(\mathrm{~s}, 3 \mathrm{H}), 2.46(\mathrm{t}, J=8.0$
$\mathrm{Hz}, 2 \mathrm{H}), 6.16(\mathrm{t}, J=7.4 \mathrm{~Hz}, 1 \mathrm{H}), 7.21(\mathrm{~d}, J=8.0 \mathrm{~Hz}, 2 \mathrm{H})$, $7.59(\mathrm{~d}, J=8.0 \mathrm{~Hz}, 1 \mathrm{H}) \cdot{ }^{13} \mathrm{C} \mathrm{NMR}\left(100 \mathrm{MHz}, \mathrm{CDCl}_{3}\right): \delta=$ 14.0, 14.1, 21.5, 22.2 22.3, 28.9, 30.8, 128.7, 129.6, 136.2, 141.2, 142.1, 144.6, 198.8. MS (EI): $m / z(\%)=230(36)$ [M+], 215 (92), 201 (20), 187 (38), 173 (29), 159 (39), 145 (22), 119 (100), 91 (71). FTIR (ATR): $v=1645$ (CO) $\mathrm{cm}^{-1}$. HRMS: $\mathrm{m} / \mathrm{z}$ calcd $\left[\mathrm{M}^{+}\right]$: 230.1665; found: 230.1662 . (E)-Methyl 4-(4-Methoxyphenyl)-3-butyl-4-oxobut-2enoate (3cb)

Hexane-EtOAc; pale yellow oil. ${ }^{1} \mathrm{H}$ NMR $(400 \mathrm{MHz}$, $\left.\mathrm{CDCl}_{3}\right): \delta=0.89(\mathrm{t}, J=7.2 \mathrm{~Hz}, 3 \mathrm{H}), 1.40-1.50(\mathrm{~m}, 4 \mathrm{H})$, $2.98(\mathrm{t}, J=7.6 \mathrm{~Hz}, 2 \mathrm{H}), 3.76(\mathrm{~s}, 3 \mathrm{H}), 3.88(\mathrm{~s}, 3 \mathrm{H}), 5.99(\mathrm{~s}$, $1 \mathrm{H}), 6.95(\mathrm{~d}, J=9.2 \mathrm{~Hz}, 2 \mathrm{H}), 7.84(\mathrm{~d}, J=9.2 \mathrm{~Hz}, 2 \mathrm{H}) .{ }^{13} \mathrm{C}$ NMR $\left(100 \mathrm{MHz}, \mathrm{CDCl}_{3}\right): \delta=13.8,22.9,29.2,30.5,51.5$, $55.5,113.8,122.0,128.8,132.2,157.9,163.9,166.1,196.5$. MS (EI): $m / z(\%)=276(28)\left[\mathrm{M}^{+}\right], 245(20), 217(22), 135$ (100). FTIR (ATR): $v=1654(\mathrm{CO}), 1722(\mathrm{COOMe}) \mathrm{cm}^{-1}$. HRMS: $m / z$ calcd $\left[\mathrm{M}^{+}\right]$: 276.1356 ; found: 276.1355 .

(E)-Methyl 4-(4-Methoxyphenyl)-3-methyl-4-oxobut-2enoate (3db)

Hexane-EtOAc; pale yellow oil. ${ }^{1} \mathrm{H}$ NMR $(400 \mathrm{MHz}$, $\left.\mathrm{CDCl}_{3}\right): \delta=2.42(\mathrm{~d}, J=1.6 \mathrm{~Hz}, 3 \mathrm{H}), 3.77(\mathrm{~s}, 3 \mathrm{H}), 3.88(\mathrm{~s}$, $3 \mathrm{H}), 6.09(\mathrm{q}, J=1.6 \mathrm{~Hz}, 1 \mathrm{H}), 6.95(\mathrm{~d}, J=9.2 \mathrm{~Hz}, 2 \mathrm{H}), 7.83$ $(\mathrm{d}, J=8.8 \mathrm{~Hz}, 2 \mathrm{H}) \cdot{ }^{13} \mathrm{C}$ NMR $\left(100 \mathrm{MHz}, \mathrm{CDCl}_{3}\right): \delta=16.0$, 51.6, 55.5, 113.9, 123.0, 128.1, 132.2, 153.1, 163.9, 166.3, 196.5. MS (EI): $m / z(\%)=234(16)\left[\mathrm{M}^{+}\right], 203(20), 135$ (100), 107 (10), 92 (10), 77 (10), 62 (9). FTIR (ATR): $v=1651(\mathrm{CO}), 1720\left(-\mathrm{COOCH}_{3}\right) \mathrm{cm}^{-1}$. HRMS: $\mathrm{m} / z$ calcd $\left[\mathrm{M}^{+}\right]$: 234.0887; found: 234.0884 .

(E)-2-Methyl-1,3-diphenylprop-2-en-1-one (3ea) Hexane-EtOAc; yellow oil. ${ }^{1} \mathrm{H}$ NMR $\left(400 \mathrm{MHz}, \mathrm{CDCl}_{3}\right)$ : $\delta=2.27(\mathrm{~d}, J=1.6 \mathrm{~Hz}, 3 \mathrm{H}), 7.18(\mathrm{~d}, J=1.6 \mathrm{~Hz}, 1 \mathrm{H}), 7.30$ $7.56(\mathrm{~m}, 8 \mathrm{H}), 7.71-7.77(\mathrm{~m}, 2 \mathrm{H}) .{ }^{13} \mathrm{C}$ NMR $(100 \mathrm{MHz}$, $\left.\mathrm{CDCl}_{3}\right): \delta=14.4,128.2,128.5,128.6,129.5,129.7,131.7$, 135.8, 136.9, 138.5, 142.2, 199.5. MS (EI): $\mathrm{m} / z(\%)=222$ (100) $\left[\mathrm{M}^{+}\right], 207$ (33), 115 (32), 77 (48). FTIR (ATR): $v=$ 1642 (CO) $\mathrm{cm}^{-1}$. HRMS: $\mathrm{m} / z$ calcd $\left[\mathrm{M}^{+}\right]: 222.1039$; found: 222.1032.

(E)-1-(4-Methoxyphenyl)-2-methyl-3-phenylprop-2-en1-one (3eb)

Hexane-EtOAc; yellow oil. ${ }^{1} \mathrm{H}$ NMR (400 $\mathrm{MHz}, \mathrm{CDCl}_{3}$ ): $\delta=2.25(\mathrm{~d}, J=1.6 \mathrm{~Hz}, 3 \mathrm{H}), 3.87(\mathrm{~s}, 3 \mathrm{H}), 6.95(\mathrm{~d}, J=9.2$ $\mathrm{Hz}, 2 \mathrm{H}), 7.10(\mathrm{~d}, J=1.6 \mathrm{~Hz}, 1 \mathrm{H}), 7.30-7.43(\mathrm{~m}, 5 \mathrm{H}), 7.81$ $(\mathrm{d}, J=9.2 \mathrm{~Hz}, 2 \mathrm{H}) .{ }^{13} \mathrm{C} \mathrm{NMR}\left(100 \mathrm{MHz}, \mathrm{CDCl}_{3}\right): \delta=14.9$, 55.5, 113.5, 128.3, 128.4, 129.6, 130.7, 132.0, 137.0, 140.0, 162.8, 198.3. MS (EI): $m / z(\%)=252(38)\left[\mathbf{M}^{+}\right], 135(100)$, 145 (15), 115 (26). FTIR (ATR): $v=1638(\mathrm{CO}) \mathrm{cm}^{-1}$. HRMS: $m / z$ calcd $\left[\mathrm{M}^{+}\right]$: 252.1145 ; found: 252.1138 . Compounds $3 \mathrm{fb}$ and $3 \mathrm{fb}^{\prime}$

Hexane-EtOAc.

(E)-2-Benzylidene-1-(4-methoxyphenyl)pentan-1-one (3fb)

Pale yellow oil. ${ }^{1} \mathrm{H}$ NMR (400 MHz, $\left.\mathrm{CDCl}_{3}\right): \delta=0.98$ (t, $J=7.2 \mathrm{~Hz}, 3 \mathrm{H}), 1.57$ (sext, $J=7.6 \mathrm{~Hz}, 2 \mathrm{H}), 2.71(\mathrm{t}, J=7.8$ $\mathrm{Hz}, 2 \mathrm{H}), 3.89$ (s, $3 \mathrm{H}), 6.95$ (d, $J=9.2 \mathrm{~Hz}, 2 \mathrm{H}), 6.98$ (s, 1 $\mathrm{H}), 7.30-7.42(\mathrm{~m}, 5 \mathrm{H}), 7.85(\mathrm{~d}, J=9.2 \mathrm{~Hz}, 2 \mathrm{H}) .{ }^{13} \mathrm{C} \mathrm{NMR}$ $\left(100 \mathrm{MHz}, \mathrm{CDCl}_{3}\right): \delta=14.3,22.0,30.2,55.5,113.5,128.1$, 128.4, 129.1, 130.9, 132.1, 135.9, 138.5, 142.4, 163.0, 198.2. MS: $(\mathrm{EI}): \mathrm{m} / z(\%)=280(56)\left[\mathrm{M}^{+}\right], 251(15), 173$ (16), 135 (100). FTIR (ATR): $v=1636\left(\right.$ CO) $\mathrm{cm}^{-1}$. HRMS: $\mathrm{m} / \mathrm{z}$ calcd $\left[\mathrm{M}^{+}\right]: 280.1458$; found: 280.1460 .

(E)-1-(4-Methoxyphenyl)-2-phenylhex-2-en-1-one (3fb') Pale yellow oil. ${ }^{1} \mathrm{H}$ NMR $\left(400 \mathrm{MHz}, \mathrm{CDCl}_{3}\right): \delta=0.92(\mathrm{t}$, $J=7.2 \mathrm{~Hz}, 3 \mathrm{H}), 1.49$ (sext, $J=7.6 \mathrm{~Hz}, 2 \mathrm{H}), 2.24(\mathrm{q}, J=7.2$ $\mathrm{Hz}, 2 \mathrm{H}), 3.85(\mathrm{~s}, 3 \mathrm{H}), 6.35(\mathrm{t}, J=7.4 \mathrm{~Hz}, 1 \mathrm{H}), 6.89(\mathrm{~d}$, $J=9.2 \mathrm{~Hz}, 2 \mathrm{H}), 7.23-7.38(\mathrm{~m}, 5 \mathrm{H}), 7.81(\mathrm{~d}, J=9.2 \mathrm{~Hz}, 2$ H). ${ }^{13} \mathrm{C}$ NMR $\left(100 \mathrm{MHz}, \mathrm{CDCl}_{3}\right): \delta=13.9,22.5,31.4,55.4$, 
$113.4,127.3,128.2,129.4,130.8,132.2,136.5,141.6$

142.2, 162.9, 196.1. MS (EI): $m / z(\%)=280(12)\left[\mathrm{M}^{+}\right], 251$ (39), 135 (100). FTIR (ATR): $v=1638(\mathrm{CO}) \mathrm{cm}^{-1}$. HRMS: $\mathrm{m} / \mathrm{z}$ calcd $\left[\mathrm{M}^{+}\right]$: 280.1458; found: 280.1460 .

(7) (a) Duke, P. J.; Boykin, D. W. Jr. J. Org. Chem. 1972, 37, 1436. (b) Black, W. B.; Lutz, R. E. J. Am. Chem. Soc. 1953, 75, 5990. (c) Mittal, S.; Durani, S.; Kapil, R. S. J. Med. Chem. 1985, 28, 492 .

(8) See Figure 1.

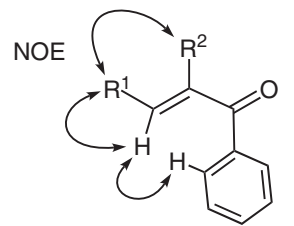

Figure 1
(9) Hayashi, T.; Inoue, K.; Taniguchi, N.; Ogasawara, M. J. Am. Chem. Soc. 2001, 123, 9918.

(10) Rhodium(I)-catalyzed reaction of 2-bromo-substituted phenylboronic acids with alkynes under $\mathrm{CO}$ atmosphere leading to indenone structures was reported. See: Harada, Y.; Nakanishi, J.; Fujihara, H.; Tobisu, M.; Fukumoto, Y.; Chatani, N. J. Am. Chem. Soc. 2007, 29, 5766. 